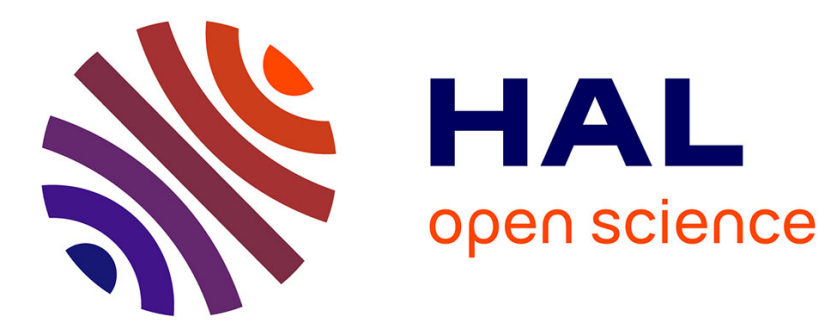

\title{
Improved GMI Sensors Using Strongly-Coupled Thin Pick-Up Coils
}

Basile Dufay, Sébastien Saez, Christophe Dolabdjian, D. Seddaoui, A. Yelon, D. Menard

\section{- To cite this version:}

Basile Dufay, Sébastien Saez, Christophe Dolabdjian, D. Seddaoui, A. Yelon, et al.. Improved GMI Sensors Using Strongly-Coupled Thin Pick-Up Coils. Sensor letters, 2009, 7 (3, Sp. Iss. SI), pp.334338. 10.1166/sl.2009.1088. hal-00988067

\section{HAL Id: hal-00988067 https://hal.science/hal-00988067}

Submitted on 7 May 2014

HAL is a multi-disciplinary open access archive for the deposit and dissemination of scientific research documents, whether they are published or not. The documents may come from teaching and research institutions in France or abroad, or from public or private research centers.
L'archive ouverte pluridisciplinaire HAL, est destinée au dépôt et à la diffusion de documents scientifiques de niveau recherche, publiés ou non, émanant des établissements d'enseignement et de recherche français ou étrangers, des laboratoires publics ou privés. 


\title{
Improved GMI Sensors Using Strongly-Coupled Thin Pick-Up Coils
}

\author{
B. Dufay ${ }^{1, *}$, S. Saez ${ }^{1}$, C. Dolabdjian ${ }^{1}$, D. Seddaoui ${ }^{2}$, A. Yelon², and D. Ménard² \\ ${ }^{1}$ GREYC UMR 6072-ENSICAEN and Université de Caen Basse Normandie, 6 Bd. Mal. Juin, France 14050 Caen Cedex \\ ${ }^{2}$ École Polytechnique de Montréal, Département de génie physique \& Regroupement québécois des \\ matériaux de pointe, CP 6079, succursale Centre-ville, Montréal, Qué., Canada H3C 3A7
}

\begin{abstract}
The performance of Giant Magneto-Impedance (GMI)-based magnetometers is currently limited by electronics, particularly as regards the equivalent magnetic noise. Thus, it is crucial to increase the sensitivity before demodulation. With that objective, we analyze one solution, using a wire-wound device, consisting of a very thin pick-up coil wrapped around a GMI wire, as a sensing element. We present an electrical sensor model using classical two-port network impedance parameters. It allows us to predict the expected improvement in sensitivity, simulating the device implementation in an appropriate electronic design. Preliminary results suggest an improvement in sensitivity by a factor 2 for a $0 \mathrm{dBm}$ sinusoidal excitation voltage source at $10 \mathrm{MHz}$. Noise measurements support this analysis and show an intrinsic output magnetic noise improvement in the same ratio.
\end{abstract}

Keywords: Magnetometer, GMI, Noise, Pick-Up Coil.

\section{INTRODUCTION}

The Giant Magneto-Impedance (GMI) effect attracts considerable attention as it appears to be an excellent candidate for the design of highly sensitive and low cost magnetometers. ${ }^{1}$ Paradoxically, few studies have investigated the noise of these devices. However, we have previously reported that the magnetic noise level of the best GMI-based sensor is dominated principally by the associated electronic conditioning noise contribution, and by intrinsic GMI wire sensitivity. ${ }^{2,3}$ Currently, the most direct way to improve measurement is to increase the sensitivity before demodulation, without added electronics noise.

With that objective, we test one solution using a wirewound device, consisting of a very thin pick-up coil wrapped around a GMI wire as a sensing element. The GMI wire used, made by MXT, ${ }^{4}$ is $1.1 \mathrm{~cm}$ long and approximately $30 \mu \mathrm{m}$ in diameter. The diameter of the 250 turn, thin, pick-up coil is $100 \mu \mathrm{m}$ and its length is $0.8 \mathrm{~mm}$. This configuration of GMI device and an associated pick-up coil, which is sometimes referred to as offdiagonal $\mathrm{GMI}^{5}$ or orthogonal fluxgate in the fundamental mode ${ }^{6}$ has previously been used in basic studies of GMI. ${ }^{7}$

${ }^{*}$ Corresponding author; E-mail: basile.dufay@greyc.ensicaen.fr
Here, we focus on the opportunities offered by the use of these coils to optimize the equivalent magnetic noise of the sensor.

First, we propose a general method to characterize the combined GMI-pick up structure and use it to analyze one example of an electronics set up. We measure an equivalent magnetic noise improvement of a factor of 2 , for the coil output.

The paper is organized as follows. The device and its two-port network impedance parameter characterization as a function of frequency are presented in Section 2 . Section 3 is dedicated to the analysis of measurement results and comparison with prediction. A general conclusion is given in Section 4.

\section{DEVICE AND ASSOCIATED ELECTRICAL MODEL}

The GMI effect is observed in ferromagnetic conductors driven by an ac current. It shows a large variation of the conductor's impedance with external applied magnetic field, due to the dependence of the effective permeability upon the applied magnetic field, inducing variation in the skin effect. The impedance depends upon the external static magnetic induction, $B_{\text {ext } \_ \text {, }}$, and upon the small 

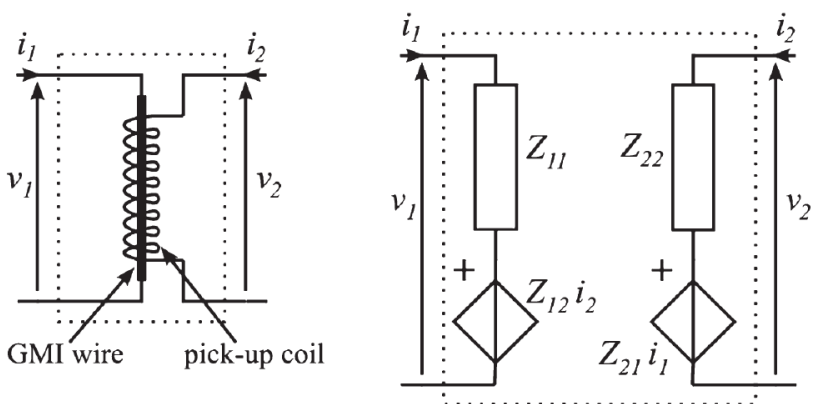

Fig. 1. Device schematic and associated electrical model, using a classical two-port network.

variation of the external, sensed, magnetic field, $\delta B_{\text {ext }}(t)$. In order to simplify the analysis, we assume that the direction of external magnetic induction $B_{\text {ext }}(t)$ and GMI sample axis are always collinear. According to a first order Taylor expansion, the complex impedance $\underline{Z}$ of a GMI sample may be written ${ }^{3}$

$$
\begin{aligned}
& \underline{Z}\left(\omega, B_{\text {ext }}(t), I_{\mathrm{dc}}\right) \\
& =\underline{Z}\left(\omega, B_{\text {ext_0}}\right)+\left.\frac{\partial \underline{Z}\left(\omega, B_{\text {ext }}, I_{\mathrm{dc}}\right)}{\partial B_{\text {ext }}}\right|_{B_{\text {ext }}=B_{\text {ext_0 }}} \delta B_{\text {ext }}(t)
\end{aligned}
$$

where $\omega$ is the angular frequency of ac driving current and $I_{\mathrm{dc}}$ is the amplitude of a dc bias current flowing through the GMI sample. The sensitivity of the sensing element is directly proportional to the term

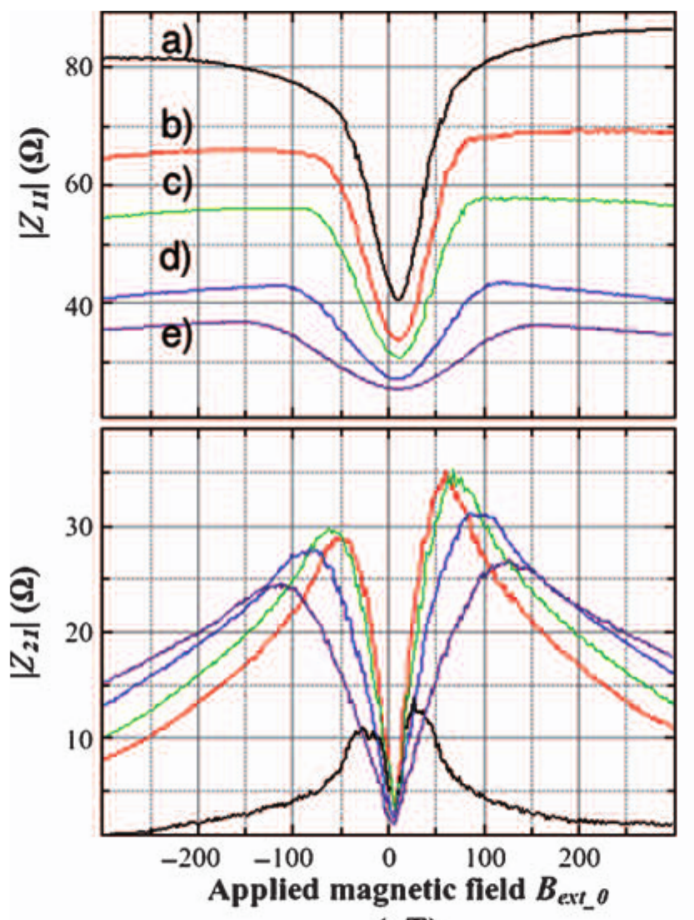

$(\mu \mathrm{T})$ $\left.\left(\partial \underline{Z}\left(\omega, B_{\text {ext }}, I_{\mathrm{dc}}\right) / \partial B_{\text {ext }}\right)\right|_{B_{\text {ext }}=B_{\text {ext } 0} \text {, which may be expressed }}$ in $\Omega / \mathrm{T}^{3}$

The expression for GMI impedance in Eq. (1) may be generalized to a GMI wire device coupled to a thin pick-up coil. The sample configuration may be modeled as a classical $z$-equivalent two-port network as illustrated in Figure 1. Each term $\underline{Z}_{i j}\left(\omega, \vec{B}_{\text {ext }}(t), I_{\mathrm{dc}}\right)$ of its equivalent matrix may be expressed following Eq. (1), leading to

$$
\begin{aligned}
& {[\underline{Z}]\left(\omega, B_{\text {ext }}(t), I_{\mathrm{dc}}\right)} \\
& \quad=\left[\begin{array}{l}
\underline{Z}_{11}\left(\omega, B_{\text {ext }}(t), I_{\mathrm{dc}}\right) \underline{Z}_{12}\left(\omega, B_{\text {ext }}(t), I_{\mathrm{dc}}\right) \\
\underline{Z}_{21}\left(\omega, B_{\text {ext }}(t), I_{\mathrm{dc}}\right) \underline{Z}_{22}\left(\omega, B_{\text {ext }}(t), I_{\mathrm{dc}}\right)
\end{array}\right]
\end{aligned}
$$

This two-port network model, associated with the well known high performance detector used by Bushida et al., ${ }^{8}$ allows us to predict any input or output, voltage or current, variations as functions of applied magnetic field. Thus, the expected performances could be deduced for several combinations of bias current, $I_{\mathrm{dc}}$, excitation voltage source frequency, $f(=\omega / 2 \pi)$, and static magnetic field working point, $B_{\text {ext } 0}$. This leads to an evaluation of the improvement expected, using a thin pick-up coil.

\section{MEASUREMENT RESULTS AND ANALYSIS}

In order to characterize the device described above, several preparatory measurements were performed, which yielded

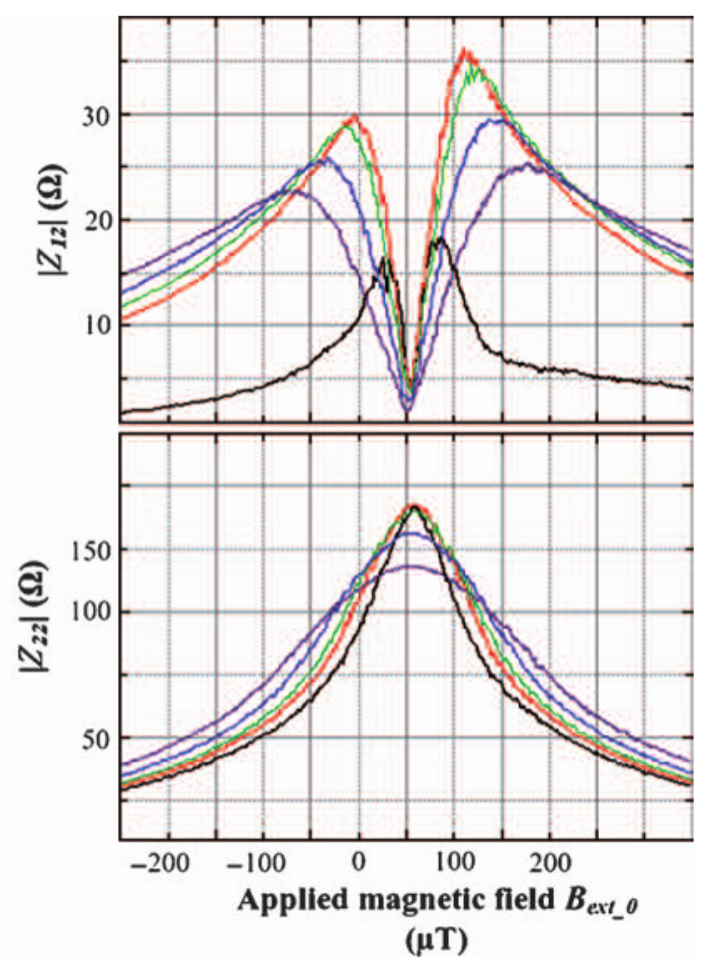

Fig. 2. Measurements of the absolute values of matrix elements $\left|\underline{Z}_{i j}\right|$ as functions of the applied magnetic field working point, at a $0 \mathrm{dBm}$ voltage excitation frequency of $10 \mathrm{MHz}$, for five bias current values: (a) $0 \mathrm{~mA}$, (b) $1 \mathrm{~mA}$, (c) $2 \mathrm{~mA}$, (d) $5 \mathrm{~mA}$ and (e) $10 \mathrm{~mA}$. 

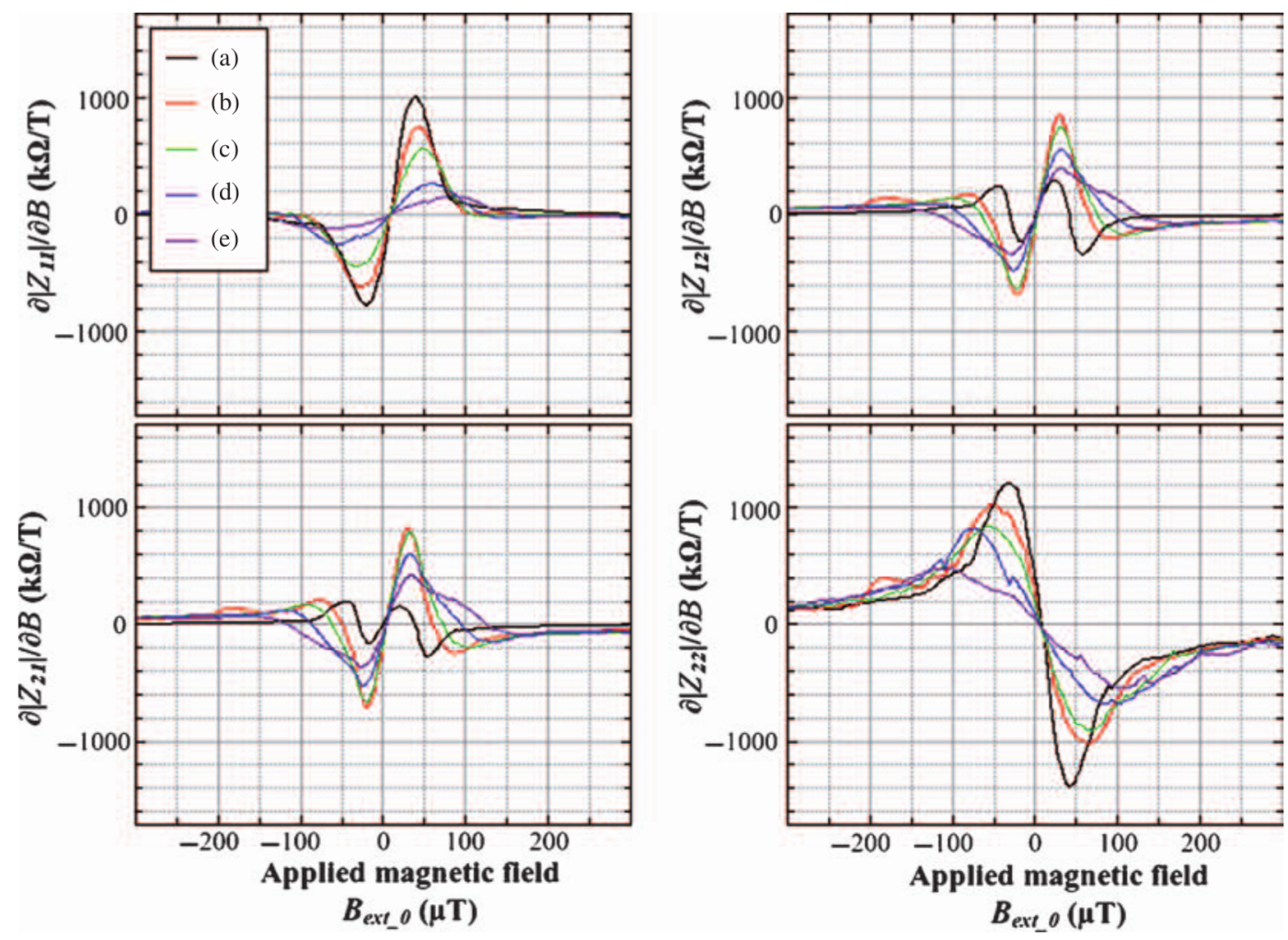

Fig. 3. Sensitivity of a two-port impedance network matrix elements as functions of applied magnetic field at a 0 dBm voltage excitation source frequency of $10 \mathrm{MHz}$ for five bias current values: (a) $0 \mathrm{~mA}$, (b) $1 \mathrm{~mA}$, (c) $2 \mathrm{~mA}$, (d) $5 \mathrm{~mA}$ and (e) $10 \mathrm{~mA}$.

classical device $S$-parameters as functions of the external applied field working point, $B_{\text {ext } 0}$, at several bias current values, $I_{\mathrm{dc}}$ (in the range of $\pm 10 \mathrm{~mA}$ ), and $0 \mathrm{dBm}$ excitation voltage source amplitude at different frequencies (in the range of $3 \mathrm{MHz}$ to $300 \mathrm{MHz}$ ). The GMI wire coupled to the thin pick-up coil was connected to an HP4396B network analyzer measuring $S$-parameters. The device was placed at the center of a solenoid applying the external

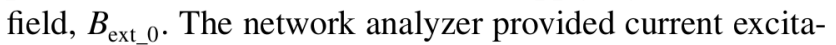
tion through wire or coil and measured the transmitted and reflected power according to each measured $S$-parameter. Bias current, $I_{\mathrm{dc}}$, and current flowing through the solenoid were provided by an external current source, HP6629A. Then, the impedance matrix parameters $[Z]$ were deduced from the $S$-parameter matrix according to the expression

$$
[Z]=z_{c}\left(\left[\begin{array}{ll}
1 & 0 \\
0 & 1
\end{array}\right]+[S]\right) \cdot\left(\left[\begin{array}{ll}
1 & 0 \\
0 & 1
\end{array}\right]-[S]\right)^{-1}
$$

where $z_{c}$ is the characteristic impedance of the line $(50 \Omega)$ and $[S]$ is the measured $S$-parameter matrix.

Figure 2 shows a few measurements of the absolute values of the matrix elements $\left|\underline{Z}_{i j}\right|$ as functions of applied

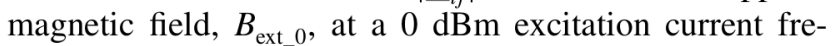
quency of $10 \mathrm{MHz}$ for five bias current values $(0,1,2$, 5 and $10 \mathrm{~mA}$ ). In generating the Figure 2, only positive bias currents are shown. As observed, the bias current reduces the amplitude of the diagonal response and creates an asymmetric off-diagonal response. Essentially the same behavior is observed for negative dc current polarization. The matrix element $Z_{11}$ represents the ratio of input voltage to input current when the output current is fixed to zero. This term is the same as the GMI impedance without a pick-up coil, as may be seen from the behavior of $\left|Z_{11}\right|$ in Figure 2. In addition, $\left|Z_{12}\right|$ and $\left|Z_{21}\right|$ are quite similar as is to be expected for passive impedance two-port networks. We note that these two terms characterize the mutual influence of input and output.

By using these results and following Eq. (1), we evaluate both $\underline{Z}_{i j}\left(\omega, B_{\text {ext } \_}, I_{\mathrm{dc}}\right)$ and $\left.\left(\partial \underline{Z}\left(\omega, B_{\text {ext }}, I_{\mathrm{dc}}\right) / \partial B_{\text {ext }}\right)\right|_{B_{\text {ext }}=B_{\text {ext } \_0}}$ as functions of static magnetic field working point $B_{\text {ext } 0}$. All device sensitivities (expressed in $\Omega / T$ ) $\partial Z_{i j} / \partial B$, presented in Figure 3, were obtained by numerical treatment of the slopes of the experimental curves. For the diagonal component, the bias current reduces the sensitivity, whereas for the off-diagonal, there is a finite $I_{\mathrm{dc}}$ which maximizes the sensitivity. Analysis of Figure 3 allows us to determine the optimal working point for each configuration: $I_{\mathrm{dc}} \approx 0 \mathrm{~mA}$, $B_{\text {ext } \_0} \approx 35 \mu \mathrm{T}$ optimize the measurement with $Z_{11}$, whereas $I_{\mathrm{dc}} \approx 1 \mathrm{~mA}, B_{\text {ext } 00} \approx 25 \mu \mathrm{T}$ should be used with $Z_{12}$ or with $Z_{21}$. Unfortunately, these optimal conditions are different. Thus, the resulting performances of the device will depend upon a combination of each of these terms, not necessarily at the greatest total sensitivity. 


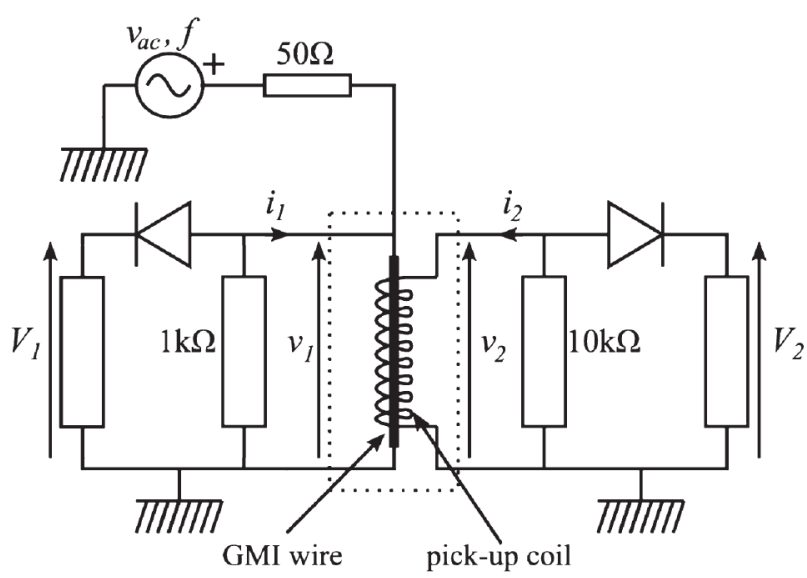

Fig. 4. Associated electronic conditioning used to measure sensitivities (in V/T) of GMI wire $T_{r_{-} w}$ and of pick-up coil $T_{r_{-} c}$.

Knowledge of the impedance matrix parameters allows us to estimate device performance when we employ electronic conditioning based on the well known high performance detector, ${ }^{3,8}$ illustrated in Figure 4. Moreover, it permits us to predict the output voltage sensitivity (in $\mathrm{V} / \mathrm{T}$ ) from measurements of the demodulated signals at the two device terminals, $V_{1}$ and $V_{2}$. In the schematic, the device is driven by a sinusoidal voltage source, $v_{\mathrm{ac}}$, of $0 \mathrm{dBm}$, which induces an ac current through the GMI

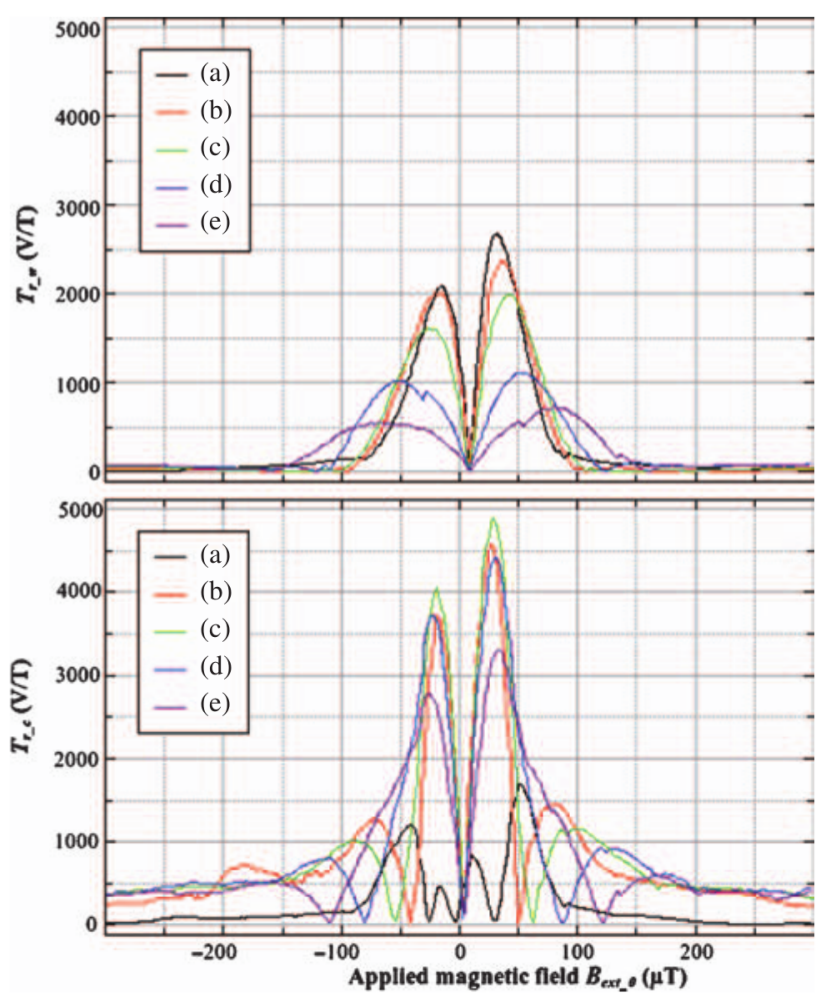

Fig. 5. Expected output device terminal sensitivities, $T_{r_{-} w}$ and $T_{r_{-} c}$, versus applied magnetic field at a $0 \mathrm{dBm}$ voltage source excitation frequency of $10 \mathrm{MHz}$ for five bias current values: (a) $0 \mathrm{~mA}$, (b) $1 \mathrm{~mA}$, (c) $2 \mathrm{~mA}$, (d) $5 \mathrm{~mA}$ and (e) $10 \mathrm{~mA}$. wire. As shown in our earlier work, driving the GMI wire with pulsed current source excitation results in better performance. Nevertheless, the implementation described here satisfies our need to validate the electrical model. This can only be done in the well understood linear regime. Induced voltages are picked up at both terminals, from diode peak detectors.

Figure 5 shows the expected resulting voltage sensitivity $T_{r-w}=\partial\left|V_{1}\right| / \partial B_{\text {ext }}$ and $T_{r-c}=\partial\left|V_{2}\right| / \partial B_{\text {ext }}$ versus applied magnetic field working point at a $0 \mathrm{dBm}$ voltage excitation frequency of $10 \mathrm{MHz}$ for five bias current values. We observe that the best sensitivity with pick-up coil (in the optimal $T_{r_{-} c}$ bias conditions, $I_{\mathrm{dc}} \approx 1 \mathrm{~mA}$ and $B_{\text {ext_0 }} \approx$ $25 \mu \mathrm{T}$ ) is twice that with GMI wire alone (also in the optimal $T_{r_{-} w}$ bias conditions, $I_{\mathrm{dc}} \approx 0 \mathrm{~mA}$ and $B_{\text {ext } \_0} \approx$ $35 \mu \mathrm{T})$. This highlights the detailed relationship between the electronics set-up and final sensitivity: all terms of the impedance matrix $[Z]$ contribute to the final sensitivity. Figure 6 summarizes the best performances anticipated with a device, as a function of the voltage excitation frequency, $f$, and of the bias current, $I_{\mathrm{dc}}$. Clearly, the pick-up coil configuration increases the sensitivity. A factor of 4 could be obtained at a frequency of $100 \mathrm{MHz}$.

Finally, we have performed noise measurements with the device driven by a high frequency pulsed current, as in our previous report. ${ }^{3,9}$ Using this electronics set up, we measured GMI device sensitivity (output $V_{1}$ ) of $5700 \mathrm{~V} / \mathrm{T}$
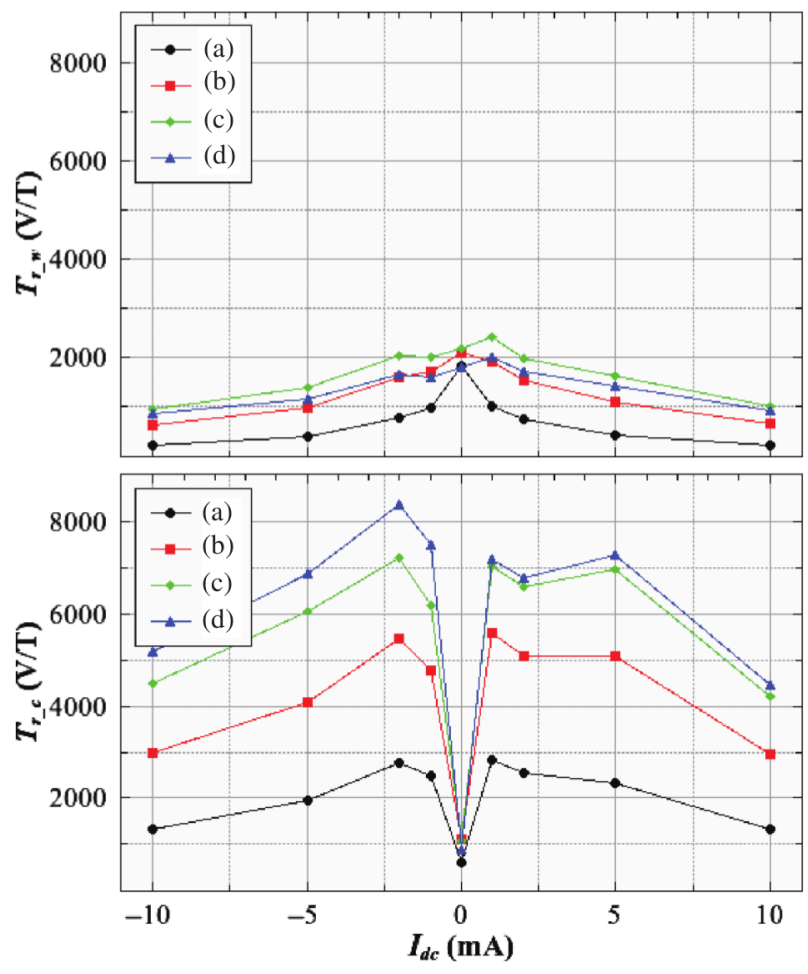

Fig. 6. Highest sensitivities, $T_{r_{-} w}$ and $T_{r_{-} c}$, according to applied magnetic field working point, $B_{\text {ext_0 }}$, for each bias current, $I_{\mathrm{dc}}$, and frequencies, $f$, at (a) $3 \mathrm{MHz}$, (b) $10 \mathrm{MHz}$, (c) $30 \mathrm{MHz}$, (d) $100 \mathrm{MHz}$. 


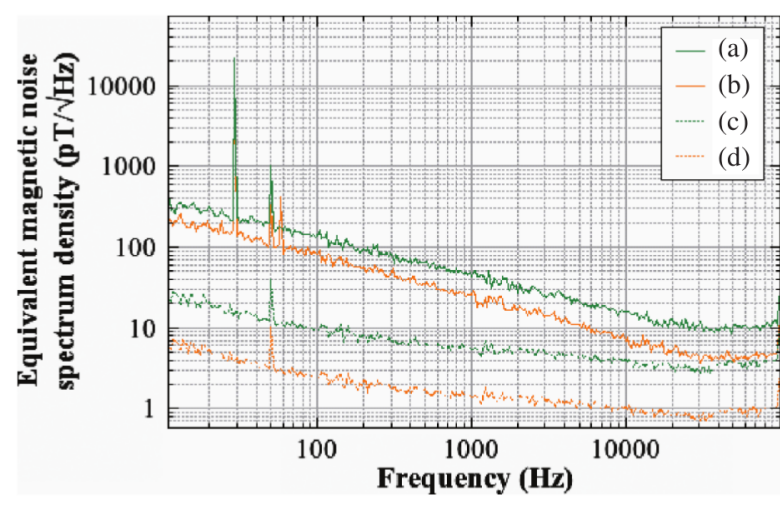

Fig. 7. Output device equivalent magnetic noise spectral densities, (a) $V_{1}(t)$ (GMI output sensitivity of $5700 \mathrm{~V} / \mathrm{T}$ ), and (b) $V_{2}(t)$ (coil output sensitivity of $22000 \mathrm{~V} / \mathrm{T}$ ). Curve (c) shows the equivalent magnetic noise limit of GMI device (voltage noise of electronics set up is divised by $5700 \mathrm{~V} / \mathrm{T}$ ). Curve (d) shows the equivalent magnetic noise limit of pick-up coil device (voltage noise of electronics set up is divised by $22000 \mathrm{~V} / \mathrm{T})$.

with its optimal bias current, and $22000 \mathrm{~V} / \mathrm{T}$ for the pickup coil (output $V_{2}$ ) also with its optimal bias current. Equivalent magnetic noise spectral densities of each output $\left(V_{1}\right.$ and $\left.V_{2}\right)$ had been measured in the GREYC shielded room and are plotted in Figure 7 (curves a and b, respectively). The same electronics voltage noise limits the output noise. The equivalent magnetic noise limitations for the $V_{1}$ and $V_{2}$ outputs, shown in Figure 7, may be calculated by the ratio of the voltage noise over the corresponding sensitivity. From the Figure 7, we see that sensitivity improvement is reflected in the sensor noise performances, which was the main goal of our investigations. By extrapolating these results to best GMI wire performance, promising results are expected for realistic sensor measurements.

\section{CONCLUSION}

We have shown that using a thin pick-up coil, wrapped around a GMI wire as a detector, is a promising approach to increasing GMI effect device sensitivity. Preliminary results show an improvement by a factor of 2 over the single wire geometry. We have proposed a modeling of such device interms of a two-port network. Further work is required to make the most out of this design. Extrapolating the results shown here to our best GMI wire performance (3 pT/Hz in Ref. [10]), promising results are expected, with GMI devices at sub $\mathrm{pT} / \sqrt{ } \mathrm{Hz}$ noise levels. ${ }^{2,3}$

Acknowledgment: This work has been partly supported by the Commission Permanente de Coopération FrancoQuébécoise.

\section{References and Notes}

1. M. Knobel, M. Vazquez, and L. Kraus, Handbook of Magnetic Materials 15, edited by K. H. J. Buschow, Elsevier (2003).

2. L. G. C. Melo, D. Ménard, A. Yelon, L. Ding, S. Saez, and C. Dolabdjian, J. Appl. Phys. 103, 033903 (2008).

3. L. Ding, S. Saez, C. Dolabdjian, L. G. C. Melo, A. Yelon, and D. Menard, IEEE Sensors Journal 9, 159 (2009).

4. MXT, Inc., Montreal, QC, Canada, http://www.m-X-t.com/.

5. S. Sandacci, D. Makhnovskiy, L. Panina, K. Mohri, and Y. Honkura, IEEE Trans. Mag. 40, 3505 (2004).

6. Z. J. Zhao, X. P. Li, J. Fan, H. L. Seet, X. B. Qian, and P. Ripka, Sens. Actuators, A 136, 90 (2007).

7. D. P. Makhnoskiy, L. Panina et, and D. J. Mapps, Phys. Rev. B 63, 144424 (2001).

8. K. Bushida, K. Mohri, and T. Uchiyama, IEEE Trans. Magn. 31-6, 3134 (1995).

9. A. Boukhenoufa, C. Dolabdjian, and D. Robbes, IEEE Sensors Journal 5, 916 (2005)

10. L. Ding, S. Saez, C. Dolabdjian, P. Ciureanu, L. G. C. Melo, A. Yelon, and D. Ménard, Sensor Letters 5, 176 (2007). 